\title{
Analysis and Comparison of SMAC and TMAC Protocol for Energy Efficient Dynamic Topology in Sensor Network
}

\author{
Tapaswini Samant, Amlan Datta
}

School of Electronics Engineering, KIIT University, India

\begin{tabular}{l} 
Article Info \\
\hline Article history: \\
Received Mar 28, 2016 \\
Revised Jul 13, 2016 \\
Accepted Jul 30, 2016 \\
\hline Keyword: \\
Energy Consumption \\
SMAC \\
TMAC \\
Wireless Sensor
\end{tabular}

\footnotetext{
Tapaswini Samant, School of Electronics Engineering, KIIT University, Bhubaneswar, Odisha, India. Email: tsamantfet@kiit.ac.in
}

Corresponding Author:

\begin{abstract}
In the era of wireless communication, wireless sensor is one of the best technologies we are witnessing. In case of environmental monitoring, tactical systems and different tracking applications, wireless sensors are being used. Here, the corresponding nodes operate on incomplete power and thus the energy comes into play to operate these entire networks. Managing the energy and its utilization is vital for TCP/IP protocol suite which is MAC layer's application. Thus keeping in mind the above challenges, the techniques used are increasing the sleep duration, over hearing and ideal listening, collision of packet and eliminating hidden terminal problem. This paper is oriented towards the comparison of energy consumption by SMAC and TMAC protocol. The characteristics of TMAC and SMAC protocols were explored keeping real transmission conditions intact, like variable transmission bit rate, dynamic topology and mobile sensors in network. TMAC and SMAC protocols are contention based protocols and are designed to keep the energy consumption low using duty cycle.
\end{abstract}

Copyright (C) 2016 Institute of Advanced Engineering and Science. All rights reserved.

\section{INTRODUCTION}

In a wireless network, multiple nodes may contend to transmit on the same shared channel at the same time. In this situation, transmitted data would get grabbed unless a suitable medium access arbitration scheme is applied. So it's the work of Medium Access Control (MAC) protocol to perform this task. The MAC protocol [1]-[4] is a sub layer of the data link layer protocol and it directly invokes the physical layer protocol. Wireless networks are mainly divided into two categories namely infrastructure based and infrastructure less wireless network. The former includes WLAN's and the second one includes mobile Adhoc networks. IEEE 802.11 standards are being used for infrastructure based wireless network and IEEE 802.3 is utilized for infrastructure less wireless network. Many MAC layer protocol for wireless networks have already been proposed, standardized and are used. MAC protocols for both infrastructure based and infrastructure less wireless network have many things in common but MAC protocol for infrastructure less wireless network is more complex since they have to address some problems that arise in the infrastructure less environment.

The main aim of the MAC protocol [5]-[9] is tantamount to enforce discipline in the access of the shared channel when multiple nodes contained to access that channel. There are other objectives of MAC protocol like maximization of utilization channel and minimization of average latency of transmission. Again, a MAC protocol must be fair and ensure that no nodes have to wait for a long time before it is allowed to transmit. 
The new generation of wireless sensor networks has various applications and distinctive challenges [10]-[12]. Few of the distinctive challenges are independent operation, limited resources and topology of a dynamic nature. Energy problem is also a challenge. So, keeping in view of the many applications and limitations of wireless sensor networks, a suitable protocol like MAC has a remarkable effect on the consumption of energy so as to prolong the lifetime of the network.

Therefore to meet the energy constraints of WSNs applications, TMAC and SMAC protocols was designed in this paper .In this paper $\mathrm{T}$ Mac and $\mathrm{S}$ Mac protocols are designed to keep the energy consumption low using duty cycle. The rest of this paper is organized as follows. We discuss the related work in section 2. In section 3 the basic MAC protocol is presented in brief. In section 4 simulation framework is explained. Section 5, simulation outcomes is explained and finally, we conclude the paper in section 6 .

\section{RELATED WORK}

A Wireless Sensor Network (WSN) is a self-organized network of tiny sensor nodes. Each sensor node (also called mote) usually senses certain physical characteristics of its environment such as temperature, sound, vibration etc. and then transmits the sensed information to the user of the network. It communicates wirelessly in a multi hop network.

Wireless sensor networks are generally battery operated so energy consumption is of great importance [12]. As a result of these critical energy features and greater network failure probability, WSN needs a well-regulated MAC protocol design. Thus, reduction of consumption of power is one of the primary goals in WSNs and it enhances the network lifetime in real time [13].

The MAC is the sub-layer of layer 2(Data link layer) which is furnishing the prospect of optimal users of controlling channel and communication channel accessed by nodes. It is likewise the primary cause of data packets to move from a particular Network Interface Card (NIC) to another throughout the shared channel.

While designing a MAC protocol there are discrete factors like throughput, latency, energy efficiency and quality of service. So, scheming a well-regulated WSN is very essential and composite problem. The function of the MAC is to help each and every node to make a decision as to when and how to access the channel. In other words, when to send a frame, listen and sleep.

\section{MEDIUM ACCESS CONTROL (MAC) PROTOCOLS}

In WSNs the nodes have to share their information through a common channel at the same time. In such situation, the information gets grabbed unless a suitable medium access arbitration scheme is applied and it is the duty of MAC protocol to carry out this task. It is the sub-layer of the data link layer protocol which provides fair access to the channels by avoiding collision. So the main aim of the MAC protocol design for an effective WSN is energy efficiency [14]-[17]. In a wireless sensor network, for a high quality MAC protocol, these attributes are to be measured.

1. Throughput: It represents how fast the amount of information is sent to the receiver through WSNs within a period of time.

2. Latency: It represents the delay i.e., how much time required for a data when sent through a network.

3. Fairness: It represents to provide fair access for all nodes by avoiding possible collision.

4. Energy Efficiency: It is an essential attribute in WSN. Since sensor nodes are battery driven it's difficult to replace or recharge batteries that's why they require energy efficient protocol design in order to prolong lifetime.

5. Collision avoidance: A collision detection scheme is difficult to implement in a wireless environment since collisions are hard to be detected by the transmission nodes. So, they have to be avoided.

6. Adaptability: This protocol needs to be adaptable for any network topology.

\subsection{Scheduled MAC protocols}

In this particular MAC protocol, the multiple access used is based on time division. Only a single node is allowed for the transmission in every time slot given. These corresponding nodes are then arranged in a clustered manner in such a way that each cluster is having a single cluster heard whose responsible is for the whole communication inside the cluster along with the other clusters nearby. The cluster head also deals with the time synchronization, time division and channel. This may create interference which may avoided by using Code Division Multiple Access (CDMA) or the Frequency Division Multiple Access (FDMA). The schedule based protocols (TDMA) have no collisions because of the single node operation per time which facilitates no idle-listening as well as no overhearing. After completion of the time slot, the node enters sleep mode. Due to lack of peer to peer connections in these protocols, results in the communication between any 
of the node only with cluster head $(\mathrm{CH})$. This shows their poor scalable and adaptable nature. For every node which leaves or joins the cluster, the $\mathrm{CH}$ every times redefines needs to synchronize whole timetable of framework inside the cluster. Due to this the $\mathrm{CH}$ gets more pressure behaves as unit exercising typical node performance. The time synchronization is maintained although the clusters face some clock drifts. Bluetooth and LEACH (Low Energy Adaptive Clustering Hierarchy).

\subsection{Event-driven protocols}

Here in these types of protocols, pre-allocation of the channels for every node is not performed considering the nodes are empty or any data to send to any other node. These event driven protocols allocate the channels based on the requirement of any individual nodes for their communication. These are thus more adaptable when any network topology changes which makes it a better protocol as compared with schedule based protocols. Changing in node density can also be maintained in event driven protocols. As in TDMA which requires time synchronization, event driven protocols are independent of it but lacks in overhearing and idle listening which was not in case of TDMA, thus leads to wastage in energy. Also some the energy is wasted is during frequency collisions while transmission of signal.

\subsection{Aloha Protocol}

The first introduced MAC protocol for general use in networks. The transmitter is independent of sending the packets on its own and without any node coordination.

\subsection{Pure Aloha Protocol}

In this protocol, without any concern with channel availability, nodes performs transmission. Here retransmission may be require as there may be the case of frequent collisions among the packets. For less collisions and low traffic these protocols are being used. For increase in traffic load, the chances of collisions increases and channel congestion occurs.

\subsection{Slotted Aloha Protocol}

This comprises the improved version of both above protocols. Here in this protocol, nodes can be transmitted in divided time slots from a channel. For the beginning of a slot to be used for transmission, nodes are in waiting position. Thus collision is avoided in transmission but may happen during the beginning of the transmission. Energy saving can be done by avoiding these collisions by using collision avoidance mechanisms. After detecting any collisions, a random back-off interval is used by the stations which avoids the subsequent collision in the next time slot during the transmission.

\subsection{Aloha with preamble sampling}

In case of this modified protocols, to listen to the channel, nodes wake up and they again go back to sleep if the channel s free until the next time slot. If the nodes identify anything then they stay awake for a valid message. Sometimes a long dummy packet is being send by the sender's node to avoid missing the wake-up schedule of neighbor which is called the "preamble". The detection of the preamble by the active neighbor node continues listening till it gets a valid data which is then confirmed with the acknowledgement frame (ACK).

\subsection{Slotted Protocols}

\subsubsection{SMAC Protocol}

SMAC or Sensor-MAC is a MAC protocol generally used in WSN (Wireless Sensors Network) for energy saving and having advantages such as collision avoidance and scalability. Due to its fixed duty-cycle which helps reducing the idle listening of the nodes. In these cases, the nodes are battery operated and a common sleep schedule is set up which is formed by the neighboring nodes.

\subsubsection{Timeout- MAC (TMAC)}

It stands for Timeout-MAC [18],[19]. It is equally a contention based MAC protocol with high energy efficiency and easy implementation. Here the duty cycle is not fixed i.e. it introduces an adaptive duty cycle. It is also designed for WSN to decrease energy consumption. S-MAC protocol's static sleep-listen periods results in lower throughput and also performs higher latency. While as in case of T-MAC protocols, when there is no activation event for a Time threshold TA, the listen periods end due to its capability to work under various traffic loads. TA decision is presented with solution for early sleeping problem of the node. As the nodes closer to the sink they must relay more traffic results in variable load in WSN. For this variable load, performance of T-MAC performs better but with the loss of broken listen periods inside the virtual clusters. 


\subsubsection{S-MAC Protocol}

It is intended for WSNs. It is designed for the purpose of saving energy in WSNs with reference to IEEE 802.11 [18],[19] MAC. It takes up various schemes to reduce wastage and consumption of energy from different sources in WSNs. It works together with a fixed duty cycle approach. It is built on the concept of 'listen/sleep mode cycle" and it is single frequency contention based protocol.

Time frame in SMAC is separated in two parts one is for sleep and the other is to listen. For a listen interval, sensor nodes are allowed to exchange information with other nodes and send a few control packets such as SYNC, RTS and CTS. The main goal of SMAC design is to minimize consumption of energy from all the various sources which are identified to be the reason behind energy wastage (collision, overhearing, over heading etc.) and increase good scalability. To achieve this there are 3 major components in SMAC.

- Periodic listen and sleep.

- Collision and overhearing avoidance.

- Message passing.

\subsection{Periodic listen and sleep:}

Each of nodes in a sensor network actually has a fixed cycle consisting of three different states, initiation, sleep, and listen. The first step, initial state, when a node is spread into the field, is to initiate and start its own schedule, and then every node go to sleep for a period of time and afterwards wake up to listen if an another node wishes to talk to it. So, cycle for sleep/listen period is repeated until it is stopped due to its battery life. For the sleep period, the node radio is turned off by the node, and determines a time to wake it later. Every node has the freedom to choose its own listen/sleep periods. However, to minimize control overhead, we prefer neighboring nodes to synchronize with each other. It means they listen all together and go to spend the night simultaneously. All neighboring nodes do not synchronize together in a multi-hop network. Nodes broadcast their schedules to exchange their own for its immediate neighbors. If there are multiple neighbors, Nodes should contend for the medium by using Request To Send (RTS) and Clear To Send (CTS) packets when the node is listening. RTS packet is first sent by the particular node, wins the medium and the receiver reply with CTS. After beginning of transmission of data, their sleep schedule does not comply with until and unless they finish their transmission. Fig. 1 shows the complete cycle of listen and sleep which is called as a frame.

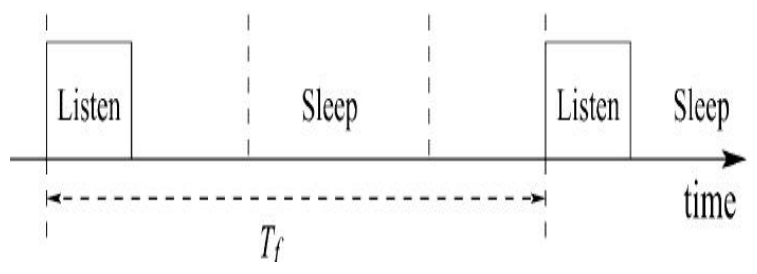

Figure 1. $\mathrm{T}_{\text {Frame }}=\mathrm{T}_{\text {listen }}+\mathrm{T}_{\text {sleep }}$

$$
\text { Duty cycle }=\mathrm{T}_{\text {listen }} / \mathrm{T}_{\text {Frame }}
$$

\subsection{Collision and overhearing avoidance:}

The nodes are able to monitor information from their direct neighboring nodes, whenever data is transmitted from node A to node B. E and F does not affect the transmission of data of A and B as E and F are out of two-hop of $\mathrm{A}$ and $\mathrm{B}$. When $\mathrm{A}$ is transmitting data to $\mathrm{B}$ and at the same time $\mathrm{D}$ wants to transmit data to $\mathrm{F}, \mathrm{B}$ will overhear the information as $\mathrm{D}$ is the direct neighbor of $\mathrm{D}$ and thus it interferes in the transmission of data from A to B. So, to avoid the collision, D must go to sleep. As C is a direct neighbor of A, C will also overhear the data that is transmitted from node A to node B. To avoid collision C has also gone to sleep. Figure 2 represents the above process.

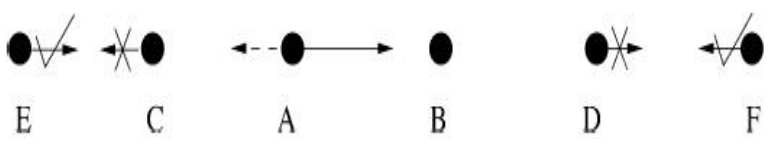

Figure 2. Neighbour nodes to go to sleep 
With reference to 802.11 MAC protocols, SMAC protocol obeys both requests to send or clear to send mechanism and virtual or physical carrier sensing mechanism. Communication course of RTS/CTS/DATA/ACK is experienced by each node when transmission of data is taken. Every packet that is being transmitted contains a domain value that shows the remaining time for the surfeit communication course. Whenever overhearing occurs during listen time in the transmitting and receiving node. The length of time is recorded meanwhile they sleep. The remaining time reduces as the time is all. If the node is in listening state and the residual time is reduced to zero. Node will be evoked. If it is not evoked then until the next listening cycle the nodes keep on sleeping.

\subsection{Message Passing}

Re-transmission is needed whenever an error occurs while transmission of a large packet. During transmission, very few bits are corrupted. So, re-transmitting the bits which are not corrupted, result in wastage of energy. Instead of that we transmit the long message fragments into a number of small independent packets, in which we have to face a longer delay and a large control overhead. SMAC restricts the medium to transmit all the fragments and convert the long message into a number of small fragments and all together sends them to a burst in order to minimize the control overhead of RTS and CTS . Each and every message and control overhead of CTS and RTS contain a domain value that indicates the leftover time for the surfeit communication. The transmission to be accurate and to introduce the neighbouring nodes, the receiving node sends an acknowledgement (ACK) for each of the received fragment. If the transmitting node does not or fails to get an acknowledgement (ACK) to the transmission time is extended for one more fragment and it transmits the same fragment immediately. Both the transmitting and receiving node record the length of time if the neighbouring node overhears the packets. In the meantime, they get some sleep. During the communication process, as time passes by, the residual time recorded will be decreased. If the node is in listening state and the residual time is reduced to zero. Node will be evoked. If it is not evoked then until the next listening cycle the nodes keep sleeping.

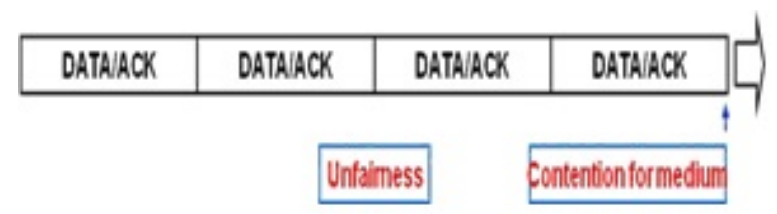

Figure 3. Message Passing

\section{SIMULATION FRAMEWORK}

In this paper, we compare the energy consumption by SMAC and TMAC protocol in different simulation scenario. We used Castalia Simulator to simulate our experiment. The Castalia simulator is based on the OMNeT++ platform. As shown in the table 1, the parameters for the framework are assumed and the comparison is shown in Figure 4 and 5.

Table 1. Simulation Scenario

\begin{tabular}{lc}
\hline \multicolumn{1}{c}{ General Parameter } & Value \\
\hline Terrain Area & $60 \times 60$ meters \\
Deployment & $5 * 5 / 7 * 7$ \\
Power Consumption per device & $0.5 \mathrm{mw}$ \\
MAC Listen interval & $10 \mathrm{~ms}$ \\
Radio Used & Telco CC2420 \\
Transmission Power & $5 \mathrm{dBm}$ \\
\hline
\end{tabular}

\section{SIMULATION OUTCOMES}

We simulated the above scenarios using Castalia Simulator. First we simulated a static network with 25 number of nodes. We observed energy consumed by SMAC and TMAC protocols in different time interval and plotted a graph using GNUPLOT. Secondly, we simulated a static network with 49 number of nodes, observed the energy consumed by SMAC and TMAC protocol and again plot a graph. 


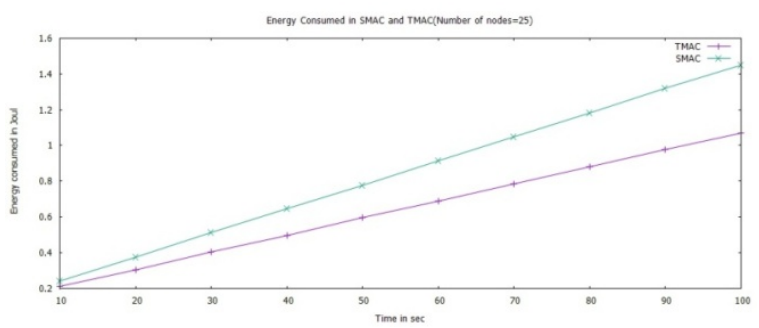

Figure 4. Energy Consumed in SMAC and TMAC in wireless sensor network with 25 number of nodes

In Figure 4, we plotted time in sec in X-axis and Energy consumed in Joule in Y-axis. In this figure we have shown the energy consumption by SMAC and TMAC protocol in a wireless sensor network with 49 number of nodes. From the Figure 5, we observed that energy consumed by SMAC is approximate $77 \%$ more than TMAC.

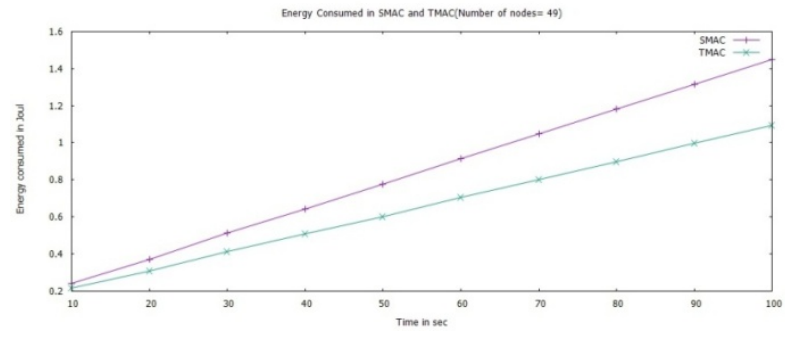

Figure 5. Energy Consumed in SMAC and TMAC in wireless sensor network with 25 number of nodes

In Figure 5, we plotted time in sec in X-axis and Energy consumed in Joule in Y-axis. In this figure we have shown the energy consumption by SMAC and TMAC protocol in a wireless sensor network with 49 number of nodes. From the figure, we observed that energy consumed by SMAC is 75\% more than TMAC.

\section{CONCLUSION}

In case of WSN, energy plays the foremost important role because of their distance constraints and high directivity, the power is the most important parameters to be taken care. Thus, the power saving in WSN is studied. In this paper, we compared two slotted MAC protocol, SMAC and TMAC with respect to their energy consumption and found that TMAC performs better than SMAC. These protocols are been studied under the same system parameters and the real time MAC layer framework setup and concluded as TMAC protocol more energy efficient than SMAC.

\section{REFERENCES}

[1] I. F. Akyildiz, et al., “A survey on sensor networks,” IEEE Communications magazine, vol/issue: 40(8), pp. 102114, 2002.

[2] M. B. Ali and M. Johnson A., "Wireless sensor networks for surveillance applications- A comparative survey of MAC protocols," Proc. The Fourth International Conference on wireless and Mobile Communications(ICWMC 2008), Athens, Greece, pp. 399-403, 2008.

[3] Durvesh P., et al., "Efficient energy saving algorithm by modern cluster head selection in Wireless Sensors Network," International Journal of Electrical and Computer Engineering, vol/issue: 3(4), pp. 167-176, 2015.

[4] Deepak S. S. and Basavaraju T. G., "Energy efficient scheme to jointly optimize coverage and connectivity in large scale wireless sensor network,” International Journal of Electrical and Computer Engineering, vol/issue: 5(3), pp. 454-463, 2015.

[5] A. Sinha and A. Chandrakasan, "Dynamic power management in wireless sensor networks,” IEEE Design \& Test of Computers, vol/issue: 18(2), pp. 62-74, 2001.

[6] Babak N. and Karim F., "Energy-efficient multi-SPEED routing protocol for wireless sensor networks," International Journal of Electrical and Computer Engineering, vol/issue: 3(2), pp. 246-253, 2013. 
[7] A. Woo and D. Culler, "A transmission control scheme for media access in sensor networks," in Proc. ACM/IEEE Int. Conf. Mobile Computing and Networking, Rome, Italy, July, pp. 221-235, 2001.

[8] B. Narain, et al., "Energy Efficient Mac Protocols For Wireless Sensor Networks: A Survey,” International Journal Of Computer Science \& Engineering Survey (IJCSES), vol/issue: 2(3), pp. 121-131, 2011.

[9] H. Jadidoleslamy, "A Survey on Medium Access Control Protocols based on Synchronous Duty Cycle Approach in Wireless Sensor Network,” IJCSNS International Journal of Computer Science and Network Security, vol/issue: 14(3), pp. 81-88, 2014.

[10] I. F. Akyildiz, et al., "Wireless sensor networks: a survey,” Computer Networks, vol/issue: 38(4), pp. 393-422, 2002.

[11] J. Kabara and M. Calle, "MAC Protocols Used by Wireless Sensor Networks and a General Method of Performance Evaluation,” International Journal of Distributed Sensor Networks, Article ID 834784, vol. 2012, pp. $1-11,2012$

[12] P. K. Pattnaik and R. Mall, "Fundamentals of Mobile Computing,” PHI Publication, 2012.

[13] P. Udhawani and S. K. Pathan, "Review of Wireless Sensor Networks and Energy Efficient MAC Protocols," International Journal of Computer Science and Information Technologies (IJCSIT), vol/issue: 5(1), pp. 868-872, 2014.

[14] R. R. Lanjewar and D. S. Adane, "Comparative Study of MAC Layer Protocols in Wireless Sensor -Networks: A Survey,” International Journal of Engineering Trends and Technology (IJETT), vol/issue: 12(1), pp. 13-19, 2014.

[15] R. Kannan, et al., "Energy and rate based MAC protocol for wireless sensor networks," Special section on sensor network technology and sensor data management, vol/issue: 32(4), pp. 60-65, 2003.

[16] Raja V., et al., "Real Time domestic power consumption monitoring using wireless sensor networks," International Journal of Electrical and Computer Engineering, vol/issue: 5(4), pp. 685-694, 2015.

[17] Umar S., et al., "Tree based energy balancing routing protocol by self-organizing in wireless sensor networks," International Journal of Electrical and Computer Engineering, vol/issue: 5(6), pp. 1486-1491, 2015.

[18] S. Agarwal, et al., "Energy Efficient MAC Protocols for Wireless Sensor Network," International Journal on Computational Sciences \& Applications (IJCSA), vol/issue: 4(1), pp. 153-160, 2014.

[19] Smritijoshi, et al., "A Novel Analysis of TMAC and SMAC Protocol for Wireless Sensor Networks Using Castalia,” International Journal of Soft Computing and Engineering (IJSCE) ISSN: 2231-2307, vol/issue: 2(6), pp. 128-131, 2013.

\section{BIOGRAPHIES OF AUTHORS}

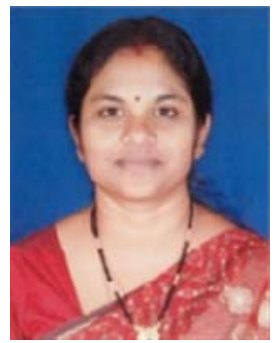

Mrs.Tapaswini Samant has done B.Tech, M.tech from KIIT University, Odisha, India. Currently she is pursuing PhD from KIIT University in Wireless Communication and her research interests includes Networking, Routing, Protocol designing. She is working as Assistant Professor in School of Electronics KIIT University since last 10 years. She has published many papers in International Conferences and participated in many national level workshops regarding her research.

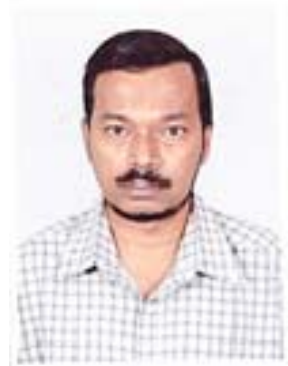

Amlan Datta has done his B. Tech, M. Tech and Ph. D from IIT, Kharagpur, India. He has worked in DRDO for nearly 5 years in Microwave and Millimeter wave. Subsequently, he has worked with Reliance Communication, India and ZTE, India in GSM, CDMA, WCDMA \& LTE for nearly 15 years. Since, last 4 years, he is working as Professor in School of Electronics Engineer, KIIT University, India. His current interests include Wireless Sensor Network, Cogni*ve Radio, Body area network and Millimeter wave communication. 